\title{
LABORATORY SIMULATION OF CYCLING OF ORGANICS IN SPACE
}

\author{
V. Mennella ${ }^{1}$
}

\begin{abstract}
Dust grains evolve during their lifetime. The chemical and physical composition of grains is determined by processes such as exposure to heat, shocks, gas, UV photons and cosmic rays. The degree of processing varies with the environments, determining different grain properties in different regions of space. In the case of organics, laboratory studies have provided a unifying interpretation of how they appear in the main phases of their evolution in space.
\end{abstract}

\section{Organics in space}

The variations of organics with the physical environments where they evolve are mainly inferred from the changes observed in the C-H stretching spectral region. The spectrum of dust in the diffuse interstellar medium is characterized by the $3.4 \mu \mathrm{m}$ absorption band (with subfeatures at about 3.38, 3.42, and $3.48 \mu \mathrm{m}$ ) and the 6.85 and $7.25 \mu \mathrm{m}$ absorption bands due to, respectively the $\mathrm{C}-\mathrm{H}$ stretching and bending modes in the methyl $\left(\mathrm{CH}_{3}\right)$ and methylene $\left(\mathrm{CH}_{2}\right)$ aliphatic groups (Sandford et al. 1991; Pendleton et al. 1994; Whittet et al. 1997; Chiar et al. 2000). The aliphatic band is absent in dense cloud spectra. In this case the $\mathrm{C}-\mathrm{H}$ stretching region is characterized by a band at $3.47 \mu \mathrm{m}$, which was assigned to the $\mathrm{C}-\mathrm{H}$ stretching mode of hydrogen (solo) bonded to tertiary $\mathrm{sp}^{3}$ carbon (Allamandola et al. 1992). An alternative attribution has been proposed by Dartois et al. (2002), who assigned the band to ammonia hydrate ice. The $3.4 \mu \mathrm{m}$ band has also been observed in solar system materials: meteorites (e.g. Cronin \& Pizzarello 1990; Ehrenfreund et al. 1991), Interplanetary Dust Particles (IDPs) (e.g. Flynn et al. 2003; Matrajt et al. 2005) and comet 81P/Wild 2 grains collected by Stardust (e.g. Keller et al. 2006; Sandford et al. 2006; Rotundi et al. 2008; Muñoz Caro et al. 2008).

\footnotetext{
1 INAF-Osservatorio Astronomico di Capodimonte, Napoli, Italy
} 


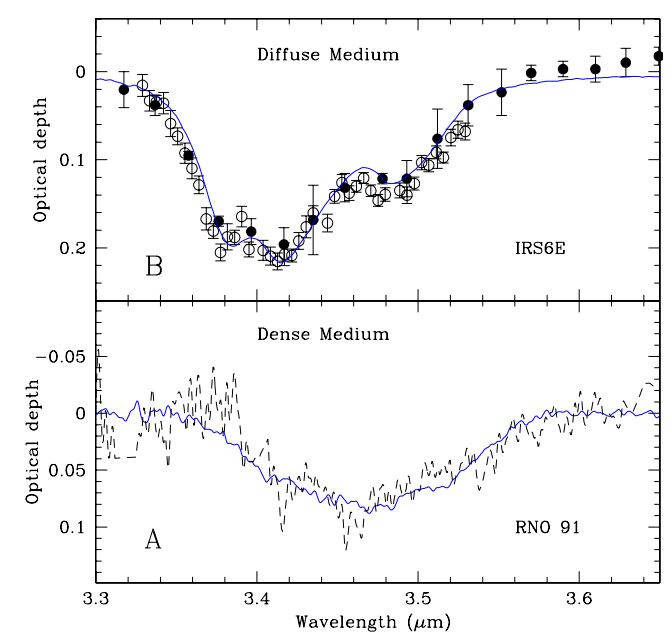

Fig. 1. A) Comparison of the $3.47 \mu \mathrm{m}$ band activated by $\mathrm{H}$ atom exposure in carbon grains covered with a water ice layer (continuous line) with that observed in a dense cloud (dashed line) (Brooke et al. 1999). B) The C-H stretching $3.4 \mu \mathrm{m}$ band of $\mathrm{H}$ processed carbon grains under simulated diffuse medium conditions (continuous line) compared to that of diffuse dust observed towards the Galactic center source IRS6E (Pendleton et al. 1994). The laboratory spectra have been scaled to match the peak optical depth of the interstellar bands.

\section{Energetic processing}

Laboratory simulation of grain processing in space has shown that the observed spectral variations of organics with various environments are the results of specific evolutionary processes. Energetic processing by UV photons and ions of aliphatic materials destroys their C-H bonds. UV irradiation of hydrogenated carbon grains and hydrocarbon molecules under simulated dense and diffuse medium conditions is indeed characterized by a strong decrease of the $3.4 \mu \mathrm{m}$ band (Mennella et al. 2001; Muñoz Caro et al. 2001). On the basis of the experimental results one can conclude that the interstellar radiation field should destroy the $\mathrm{C}-\mathrm{H}$ bonds aliphatic component of diffuse regions in a very short time scale ( $10^{4}$ years).

A similar destruction of the aliphatic C-H bonds is observed during $30 \mathrm{keV} \mathrm{He}^{+}$ irradiation of hydrogenated carbon grains under simulated dense and diffuse interstellar medium conditions. Mennella et al. (2003) concluded that cosmic rays can destroy $\mathrm{C}-\mathrm{H}$ bonds in dense clouds during the dense cloud life time of $3 \times 10^{7}$ years. Recently, Godard et al. (2011) (see also this volume) have irradiated hydrogenated carbon materials with 7 ion species and concluded that cosmic rays are not efficient enough to completely dehydrogenate the $3.4 \mu \mathrm{m}$ band carriers during the cloud lifetime. The different conclusions depend on the different approximations and assumptions adopted to estimate the effects of cosmic rays on the interstellar 


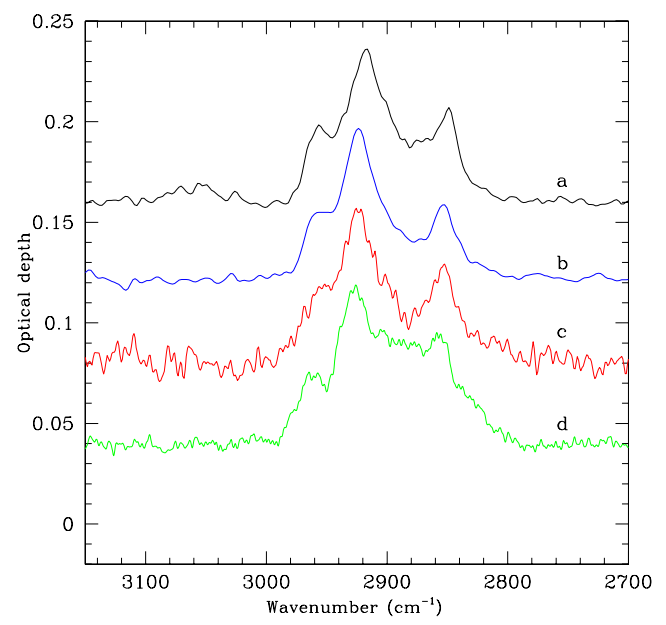

Fig. 2. The $3.4 \mu \mathrm{m}$ band of the Stardust particles C2054,0,35,16,0 (Keller et al. 2006) (a), and C2054,0,35,81,0 (Muñoz Caro et al. 2008) (b) and of the IDP L2036R9 (Muñoz Caro et al. 2006) (c) compared with that of carbon grains at room temperature after $\mathrm{H}$ atom processing under simulated dense cloud conditions (d). The spectra are scaled to the peak intensity of asymmetric $\mathrm{CH}_{2}$ modes and shifted for clarity.

aliphatic materials, starting from the experimental results. However, if the same assumptions on the cosmic ray flux, on the UV flux produced by cosmic rays and on the model adopted to estimate the destruction cross section from the laboratory data are used, the two methods give results in good agreement (Mennella et al. 2012 , in preparation). It is possible to conclude that in dense clouds cosmic rays lower the hydrogenation degree of the carrier of the aliphatic feature below the upper limit estimated by Muñoz Caro et al. (2001).

\section{$3 \mathrm{H}$ atom irradiation}

Simulation of carbon dust processing under diffuse and dense medium conditions indicates that the key process for the evolution of the interstellar aliphatic carbon component is its interaction with $\mathrm{H}$ atoms. This interaction is able to counteract the destruction of $\mathrm{C}-\mathrm{H}$ bonds by $\mathrm{UV}$ photons and cosmic rays and it activates the $3.4 \mu \mathrm{m}$ band in diffuse interstellar regions and the $3.47 \mu \mathrm{m}$ feature in dense clouds (see Fig. 1) (Mennella et al. 2002; Mennella 2008). The interesting aspect is that the same carbon grain population can absorb at the two wavelengths as a consequence of evolutionary transformations caused by processing. The transformations are compatible with the time-scale required by fast cycling of materials between dense and diffuse regions of the ISM.

Moreover, this carbon component, after the inclusion in a comet during the formation process in the cold outer edge of the solar nebula, may evolve to develop 
the $\mathrm{CH}_{2}$ and $\mathrm{CH}_{3}$ groups (as suggested by laboratory simulations, see Fig. 2) and contribute to the aliphatic band at $3.4 \mu \mathrm{m}$ of IDPs and particles of comet Wild 2 collected by the Stardust mission (Mennella 2010).

This work has been supported by ASI research contracts.

\section{References}

Allamandola, L.J., Sandford, S.A., Tielens, A.G.G.M., \& Herbst, T.M., 1992, ApJ, 399, 134

Brooke, T.Y., Sellgren, K., \& Geballe T.R., 1999, ApJ, 517, 883

Chiar, J.E., Tielens, A.G.G.M., Whittet, D.C.B., et al., 2000, ApJ, 537, 749

Cronin, J.R., \& Pizzarello, S., 1990, Geochim. Cosmochim. Acta, 54, 2859

Dartois, E., d'Hendecourt, L., Thi, W., Pontoppidan, K.M., \& van Dishoeck, E.F., 2002, A\&A, 394, 1057

Ehrenfreund, P., Robert, F., D'Hendencourt, L., \& Behar, F., 1991, A\&A, 252, 712

Flynn, G.J., Keller, L.P., Feser, M., Wirick, S., \& Jacobsen, C., 2003, Geochim. Cosmochim. Acta, 67, 4791

Godard, M., et al., 2011, A\&A, 529, 146

Keller, L.P., et al., 2006, Science, 314, 1728

Matrajt, G., Muñoz Caro, G.M., Dartois, E., D’Hendecourt, L., Deboffle, D., \& Borg, J., 2005, A\&A, 433, 979

Mennella, V., 2010, ApJ, 718, 867

Mennella, V., 2008, ApJ, 682, L101

Mennella, V., Baratta, G.A., Esposito, A., Ferini, G., \& Pendleton, Y.J., 2003, ApJ, 587, 727

Mennella, V., Brucato, J.R., Colangeli, L., \& Palumbo, P., 2002, ApJ, 569, 531

Mennella, V., Muñoz Caro, G., Ruiterkam, R., et al., 2001, A\&A, 367, 355

Muñoz Caro, G.M., Dartois, E., \& Nakamura-Messenger, K., 2008, A\&A, 485, 743

Muñoz Caro, et al., 2006, A\&A, 459, 147

Muñoz Caro, G., Ruiterkam, R., Schutte, W.A., Greenberg, J.M., \& Mennella, V., 2001, A\&A, 367, 347

Pendleton, Y.J., Sandford, S.A., Allamandola, L.J., Tielens, A.G.G.M., \& Sellgren, K., 1994, ApJ, 437, 683

Rotundi, A., et al., 2008, Meteoritics Planet. Sci., 43, 367

Sandford, S.A., Allamandola, L.J., Tielens, A.G.G.M., Sellgren, K., Tapia, M., \& Pendleton, Y., 1991, ApJ, 371, 607

Sandford, S.A., et al., 2006, Science, 314, 1720

Whittet, D.C.B., et al., 1997, ApJ, 490, 729 